\title{
Coherent network detection of gravitational waves: the redundancy veto
}

\author{
Linqing Wen and Bernard F Schutz \\ Max Planck Institut fuer Gravitationsphysik, Albert-Einstein-Institut, Am Muehlenberg 1, \\ D-14476 Golm, Germany
}

Received 14 April 2005, in final form 3 August 2005

Published 6 September 2005

Online at stacks.iop.org/CQG/22/S1321

\begin{abstract}
A network of gravitational wave detectors is called redundant if, given the direction to a source, the strain induced by a gravitational wave in one or more of the detectors can be fully expressed in terms of the strain induced in others in the network. Because gravitational waves have only two polarizations, any network of three or more differently oriented interferometers with similar observing bands is redundant. The three-armed LISA space interferometer has three outputs that are redundant at low frequencies. The two aligned LIGO interferometers at Hanford WA are redundant, and the LIGO detector at Livingston LA is nearly redundant with either of the Hanford detectors. Redundant networks have a powerful veto against spurious noise, a linear combination of the detector outputs that contains no gravitational wave signal. For LISA, this 'null' output is known as the Sagnac mode, and its use in discriminating between detector noise and a cosmological gravitational wave background is well understood. But the usefulness of the null veto for groundbased detector networks has been ignored until now. We show that it should make it possible to discriminate in a model-independent way between real gravitational waves and accidentally coincident non-Gaussian noise 'events' in redundant networks of two or more broadband detectors. It has been shown that with three detectors, the null output can even be used to locate the direction to the source, and then two other linear combinations of detector outputs give the optimal 'coherent' reconstruction of the two polarization components of the signal. We discuss briefly the implementation of such a detection strategy in realistic networks, where signals are weak, detector calibration is a significant uncertainty, and the various detectors may have different (but overlapping) observing bands.
\end{abstract}

PACS numbers: $04.80 . \mathrm{Cc}, 04.80 . \mathrm{Nn}$, 95.55.Ym, 95.85.Sz

(Some figures in this article are in colour only in the electronic version) 


\section{Introduction}

We are entering a new phase in the development of gravitational wave astronomy. The new generation of large-scale gravitational wave $(\mathrm{GW})$ interferometers has begun operating. In particular, the American LIGO detectors [3] are very close to their first sensitivity goals, having surpassed the older generation of cryogenic bar detectors [21] in sensitivity to almost all potential sources in a broad intermediate frequency band, from a few tens of $\mathrm{Hz}$ to several kilohertz. LIGO and its German-British partner GEO600 [4] have taken data so far in four 'science runs', sometimes also in cooperation with the Japanese TAMA detector [5]. (For upper limits from the earliest of these runs, see [7-11].) The Italian-French VIRGO detector [6] is currently undergoing commissioning. By the end of 2005, LIGO and GEO are expected to have embarked on full-time observing. Within a year or two we should know whether or not the first-stage sensitivity of these detectors is sufficient to make the first detections of gravitational waves, or whether the field will have to wait for the sensitivity upgrades that are planned over the subsequent five years.

The principal problem facing GW detection is distinguishing weak signals from detector noise. The main sources of noise in detectors ideally produce Gaussian (normally-distributed) amplitude noise, but all detectors have poorly understood sources of 'non-Gaussian noise', characterized by large-amplitude disturbances that occur much more frequently than in a Gaussian distribution. These disturbances are unpredictable and it is often difficult, if not impossible, to trace them to their cause, which may be external to the detector or the result of a temporary malfunction within the detector. Genuine GW signals, of course, are also expected to be rare, so they can in principle be confused with non-Gaussian noise events. Even if the waveform of an expected signal is known ahead of time, there is some chance that noise will match it well enough to confuse.

The optimal way to detect signals with a network of detectors that have ideal Gaussian noise backgrounds is called 'coherent detection', and has been studied by a number of authors [13-16]. If the direction to a source is known, then one forms linear combinations of the detector outputs, with suitable time delays, which best give the amplitudes of the two independent gravitational-wave polarizations. These superposed data streams can be studied for signals using matched filtering or less specific methods, like wavelet transforms or timefrequency methods. Gravitational waves are identified if these superposed streams contain excursions that would have very low probability in the purely Gaussian noise of the detectors.

Unfortunately, this coherent method is vulnerable to confusion through a non-Gaussian noise event in a single detector, which could make an unexpectedly large excursion in the superpositions that represent the GW polarizations. Therefore, current searches use coincidence testing, where individual data streams must each pass an amplitude threshold (usually after processing the data stream in some way); only coincident events are taken to be candidates for GWs [7, 17]. This cuts down the confusion from non-Gaussian noise, since these relatively rare noise events do not often occur at the same time (or with suitable time delays for the wave travel time) in different detectors. But the coincidence method is clearly non-optimal for a general network of detectors, since the quadrupole antenna patterns of differently oriented detectors will respond differently to a real GW, so that in some cases the detector outputs will not in fact all cross the pre-defined amplitude threshold in coincidence, and the wave will not be recognized (a false dismissal). What is more, a threshold criterion on its own is a crude identifier for a GW: it does not use all the information about the waveform that is present in the data streams, and so it can lead to false detections. More sophisticated analysis methods based on phase correlation of triggered events from different detectors can be found in $[18,19]$. 
We discuss here a simple analysis method designed to protect the coherent method from confusion by spurious noise. First introduced by Gürsel and Tinto [12] in 1989, it works in a network of detectors that contains enough information to construct what we call a null stream, by which we mean a particular linear combination of all the available data that cancels out a GW signal from a particular sky direction. We call such networks redundant, since they contain enough information to reconstruct the response of one detector from the responses of the others, regardless of the polarization of the wave. The null stream in a redundant network is a consistency check to test whether a candidate GW event is produced by detector noise or by a real GW. If the null stream shows an unusual excitation, then the candidate should be rejected (vetoed), since only noise can excite the null stream. Conversely, if individual detectors register an excitation that is absent from the null stream, then it is very likely to be a real gravitational wave, since cancellation in the null stream of a noise-generated event would require a highly improbably coincidence in amplitude and phase among all the detectors in the network. In practice, all current and planned networks of detectors are redundant or nearly so, which means that the null-stream test can be implemented as a veto in upcoming realistic searches.

Crucially, the null stream provides a model-independent veto, depending only on the description of GWs provided by general relativity. (We will discuss briefly the way in which the null stream can actually be used to test the GW model of general relativity.) The null stream does not require a source model, an assumption about polarization, or a transfer function that shows how a particular disturbance can affect the data stream of a detector. If the network is redundant, then the null stream constructed for a particular source direction cannot contain any signal from sources in that direction. This veto can be used to supplement thresholding in a coincidence search. This method is different from cross-correlation methods in that it checks the consistency in both the amplitude and phase. We expect that it can dramatically reduce the number of false identifications.

For the three-armed LISA space interferometer, the use of the 'null' output in discriminating between detector noise and a cosmological gravitational wave background is well understood $[1,2]$. But the usefulness of the null veto for ground-based detector networks has been ignored until now. Gürsel and Tinto argue that the null stream can be used to identify the locations of sources on the sky (and therefore the polarizations of GWs) in the case of three detectors at different sites, since it will consist of pure noise only when constructed for the correct source location. We have performed simulations that bear this out.

In practice, the null stream veto has limitations. For example, detectors in a redundant network may have non-overlapping bandwidths, or one of the detectors may be significantly less sensitive than the others. In these cases the null stream is self-limiting: it will contain so much noise that a GW will not be visible, making the stream useless. For this reason the null stream is unlikely to be useful for networks of bar detectors, or in networks where there are big differences in detector sensitivity. Moreover, the null stream is sensitive to calibration errors, and calibrating gravitational wave detectors is difficult. Therefore residuals of real signals may remain in the null stream at the ten per cent level, which is the typical calibration uncertainty at present.

Implementing the null-stream veto essentially means rejecting events if the null stream contains an unexpectedly large excitation, which must be due to 'non-Gaussian' noise events in one or more detectors. This imposes the requirement that a $\mathrm{GW}$ can only be identified with confidence if the event arrives at a moment when all the detectors are operating in their 'normal' state, i.e. with purely Gaussian noise (after removal of known instrumental artefacts, e.g., lines). Since non-Gaussian events are rare, and GW events are even more rare, it seems safe to impose this requirement. Indeed, it is difficult to see how a first GW detection could 
be claimed with any confidence if it were known that at least one of the detectors itself had a simultaneous non-Gaussian noise event. It follows that a crucial implementation issue for the null-stream veto is the choice of a criterion for whether the null stream is consistent with Gaussian noise at the time of an event. In this paper we use a chi-squared test for our simulations, but the actual test may depend on the kind of processing of the individual detector streams that took place before the 'event' was recognized.

In this paper, we explore the null-stream veto and detector for unknown waveforms (i.e., the so-called burst GWs) observed by interferometers, although the veto could be applied to any type of signal. We consider three realistic cases: the two LIGO Hanford detectors (the $4 \mathrm{~km} \mathrm{H1}$ and the $2 \mathrm{~km} \mathrm{H} 2$ ), which form a redundant network of just two detectors; a three-detector redundant network consisting of H1, the LIGO Livingston detector (L1), and GEO600; and an approximately redundant two-detector network consisting of H1 and L1. In section 2, we discuss the principle of constructing a null stream for a network of two and three detectors. In section 3, we propose a method for the consistency check of the 'absence' of GW signals in the null stream. In section 4 , we show a few examples using simulated data to demonstrate the performance of our approach. In section 5, we discuss the possible applications of our method and future work.

\section{Principle of null data stream construction}

The strain created in the $i$ th interferometric detector of a network by a GW arriving from a sky direction given by the right ascension and declination angles $\alpha$ and $\delta$ is a linear combination of the two polarizations of the wave,

$$
h_{i}(t)=f_{i}^{+}(t, \alpha, \delta) h_{+}(t)+f_{i}^{\times}(t, \alpha, \delta) h_{\times}(t),
$$

where $t$ is the time at the detector, $f_{i}^{+}(t, \alpha, \delta)$ and $f_{i}^{\times}(t, \alpha, \delta)$ are the detector's antenna beam pattern functions (or response) to the plus and cross polarizations of the waves, and $h_{+}$and $h_{\times}$are the amplitudes of the two polarizations of the GW. The definition of the ' + ' and ' $\times$' polarizations depends on an arbitrary orientation angle $\psi$ defined on the sky [20], but does not depend on detector orientation. The antenna beam patterns are explicit functions of time because the detector will normally change its orientation with time. In this paper, we will use as a reference time the local clock time at one of the detectors in the network (L1 when it is included in the network). For other applications, such as for pulsar observations, it might be more robust to adopt the solar barycentre as the time reference.

Following the coordinate system adopted in [20], we can rewrite

$$
f_{i}^{+}=A_{i} \sin \left(2 \psi+\xi_{i}\right), \quad f_{i}^{\times}=A_{i} \cos \left(2 \psi+\xi_{i}\right)
$$

where $A_{i}=\sqrt{f_{i}^{+2}+f_{i}^{\times 2}}$ is the amplitude of the antenna beam pattern, directly proportional to the observed amplitude of the wave, and where $\xi_{i}=\tan ^{-1}\left(f_{i}^{+} / f_{i}^{\times}\right)$at $\psi=0$ is a quantity that describes the detector's different response to the two polarizations and therefore depends on detector orientation. Equation (1) can then be re-written as

$$
h_{i}(t)=A_{i} \sqrt{h_{+}^{2}(t)+h_{\times}^{2}(t)} \sin \left(2 \psi+\xi_{i}+\xi^{h}(t)\right),
$$

where $\xi^{h}(t)=\tan ^{-1}\left(h_{\times}(t) / h_{+}(t)\right)$ is the effective phase of the waves, and is a quantity intrinsic to the wave. For a circularly polarized GW of monotonic frequency $f, \xi^{h}(t)=2 \pi f t$.

For a given incoming GW, the observed strains can therefore be different from detector to detector in two respects. (1) The measured amplitude of the wave is directly proportional to the amplitude of the detector's antenna beam pattern $A_{i}$ for the wave's direction. (2) The observed effective wave phase (therefore the apparent wave arrival time, which is normally 
referred to a fiducial feature in the waveform) is shifted by $\xi_{i}$, which depends on the detector orientation. Importantly, for an incoming wave of dominant frequency $f_{0}$, the difference between the geometrical time delay (wave arrival-time difference between detectors) and the measured delay can be as large as $1 /\left(2 f_{0}\right)$, e.g. $2 \mathrm{~ms}$ for $\mathrm{GW}$ with $f_{0} \sim 250 \mathrm{~Hz}$. This can be significant, given that the maximum geometrical time delays between currently operating detectors are between 10 and $30 \mathrm{~ms}$. Note that both quantities $A_{i}$ and $\xi_{i}$ are independent of the wave polarization angle $\psi$.

In the following two subsections, we discuss how to construct the null data stream for particular networks of two and three detectors.

\subsection{Two detectors at the same site: $H 1-H 2$}

The LIGO detectors at the Hanford site consist of two interferometers (H1 and H2), 4 and $2 \mathrm{~km}$ long, sharing the same vacuum system. The antenna patterns of these detectors are identical, and it follows that the GW strain outputs of the two detectors, $h_{1}(t)$ and $h_{2}(t)$, as given by equation (1), are identical. The null stream $N_{2}(t)$ in this case is therefore particularly simple:

$$
N_{2}(t)=h_{1}(t)-h_{2}(t) .
$$

Any GW signal will cancel in $N_{2}$, up to calibration errors, while independent noise in the detectors will not. In this simple case it is not even necessary to assume a direction for the incoming GW.

Since the detectors share the same vacuum system, some noise sources may be correlated in these two interferometers. An important motivation for making $\mathrm{H} 2$ half the length of $\mathrm{H} 1$ in the LIGO design [22] was to discriminate against highly correlated disturbances, those that might move the mirrors of both interferometers by similar amounts. Such a disturbance would produce a signal strain twice as large in $\mathrm{H} 2$ as in $\mathrm{H} 1$, and this would indicate that the disturbance was not a gravitational wave. Thus, the null-stream veto was built into LIGO from the start.

Any larger network containing $\mathrm{H} 1$ and $\mathrm{H} 2$ could use $N_{2}$ as a veto. We will explore vetos in more general networks, however, since $\mathrm{H} 1$ and $\mathrm{H} 2$ alone do not return polarization or direction information about the source.

\subsection{Three independent detectors at different sites}

We now discuss how to construct the null stream using data from three detectors at different sites (e.g., L1, H1, GEO). Because of the curvature of the Earth, such detectors cannot have exactly coincident antenna patterns. The time series of the GW strain plus noise observed by detectors 1-3 can be written as a set of three linear equations (the same as equations (4.1a, b, c) of [12]),

$$
\begin{aligned}
& h_{1}(t)=f_{1}^{+}(t) h_{+}(t)+f_{1}^{\times}(t) h_{\times}(t)+n_{1}(t) \\
& h_{2}\left(t_{2}\right)=f_{2}^{+}\left(t_{2}\right) h_{+}(t)+f_{2}^{\times}\left(t_{2}\right) h_{\times}(t)+n_{2}\left(t_{2}\right) \\
& h_{3}\left(t_{3}\right)=f_{3}^{+}\left(t_{3}\right) h_{+}(t)+f_{3}^{\times}\left(t_{3}\right) h_{\times}(t)+n_{3}\left(t_{3}\right),
\end{aligned}
$$

where we define

$$
t_{2}=t+\tau_{12}, \quad t_{3}=t+\tau_{13}
$$

and where in turn $\tau_{1 i}$ is the geometrical wave arrival time delay expected in detector $i$ with respect to detector 1 . We have shown explicitly the dependence of the antenna pattern functions 
$f_{i}^{+, \times}$on the measurement time of the wave at the moving antenna, but we have not shown the implicit source-direction angular dependence in $\tau_{12}, \tau_{13}, h_{i}$ and $f_{i}^{+, \times}$in order to keep the expressions concise. The intrinsic wave amplitudes $h_{+}$and $h_{\times}$are all evaluated at the same time argument $t$, without time delays, because we want to compare the excitation of the various detectors by the same part of the incoming wave form; strictly one should regard the time argument of $h_{+, \times}$as the retarded time along the wave's direction of travel. We also include explicitly here the noise $n_{i}$ at the appropriate measurement time in detector $i$. In practice, we require that the noise be stationary and Gaussian within a time scale much longer than the duration of the triggered events (typically tens of milliseconds for burst GW sources).

It is clear just from counting variables that these three equations admit a linear combination that cancels out the GW amplitudes $h_{+}$and $h_{\times}$completely (equation (4.2) of [12]). The null stream for three detectors is then

$$
N_{3}(\alpha, \delta, t)=A_{23} h_{1}(t)+A_{31} h_{2}\left(t+\tau_{12}\right)+A_{12} h_{3}\left(t+\tau_{13}\right),
$$

where

$$
A_{i j}(\alpha, \delta, t)=f_{i}^{+}\left(t_{i}, \alpha, \delta\right) f_{j}^{\times}\left(t_{j}, \alpha, \delta\right)-f_{j}^{+}\left(t_{j}, \alpha, \delta\right) f_{i}^{\times}\left(t_{i}, \alpha, \delta\right),
$$

and where we adopt the convention that $t_{1}=t$ the measurement time at our reference detector 1 . We see that $A_{i j}$ is a function of the source direction $(\alpha, \delta)$ and of time (due to the motion of the detectors), but it is independent of the polarization angle $\psi$. Note that, using the variables introduced in equation (2), an alternative and somewhat simpler expression for $A_{i j}$ is

$$
A_{i j}(\alpha, \delta, t)=A_{i} A_{j} \sin \left(\xi_{i}-\xi_{j}\right)
$$

where of course $A_{i}$ and $\xi_{i}$ depend on $\alpha, \delta$ and $t$.

Note that if two of the three detectors, say 1 and 2, are collocated and perfectly aligned, as is the case for $\mathrm{H} 1$ and $\mathrm{H} 2$, then the coefficient $A_{12}$ will vanish while $A_{23}=-A_{31}$. This means that $N_{3}$ becomes simply proportional to $N_{2}$, and the three-detector case degenerates to our previous two-detector example. More generally, if the source direction is such that one of the $A_{i j}$ coefficients is small, which means that the corresponding detectors have nearly the same response to the wave, then the expression automatically reduces the contribution of the third detector to the veto.

\subsection{Two detectors with nearly-aligned antenna patterns}

Among currently operating GW detectors, the antenna patterns of L1 and H1 have been designed to be aligned as closely as possible with each other, given the curvature of the Earth between them. The sky directions of their maximum sensitivity $\left(A_{i}\right)$ are offset by $\sim 25^{\circ}$ (figure 1). The two detectors are not exactly redundant, as are $\mathrm{H} 1$ and $\mathrm{H} 2$, but the concept must hold in some approximation. We consider here the possibility of constructing a 'nearly null' data stream for two nearly perfectly aligned detectors (i.e., where $A_{12} \sim 0$ ).

The residual SNR remaining in any constructed 'nearly' null stream unavoidably depends on signal waveforms. Therefore, the 'best' null-stream construction method is also waveformdependent in general. In this paper, we consider specifically constructing the nearly null stream based on a simple linear combination of data streams from L1 and H1,

$$
Q(\alpha, \delta, t)=A_{2} h_{1}(t)+v A_{1} h_{2}\left(t+\tau_{12}\right)
$$

where $v$ is a constant depending on source direction only. We choose $v$ by minimizing the waveform-independent coefficient in the rms amplitude of the residual signal $\sqrt{\sum_{t} Q^{2}(\alpha, \beta, t)}$ over a duration of time $T$. This yields, in the notation of equation (2),

$$
v=-\cos \left(\xi_{1}-\xi_{2}\right)
$$




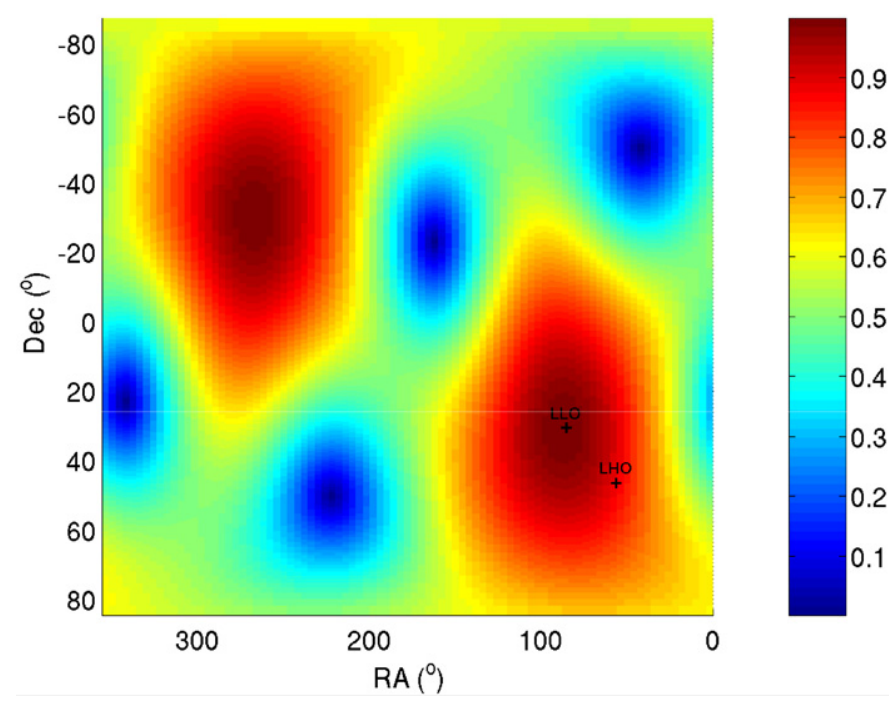

Figure 1. The all-sky map of the amplitude of the antenna beam pattern $\left(A_{1}\right)$ of LIGO Livingston (L1) at an arbitrary time of $0.00 \mathrm{hr}, 18 \mathrm{March} 2004$. The locations for L1 and H1 are labelled as 'LLO' and 'LHO' and marked with symbols ' + '.

and the rms amplitude of the residual signal in the null stream is

$$
\left|\sin \left(\xi_{1}-\xi_{2}\right)\right| A_{1} A_{2} \sqrt{\sum_{t}\left(h_{+}^{2}(t)+h_{\times}^{2}(t)\right) \cos ^{2}\left(2 \psi+\xi^{h}(t)+\xi_{2}\right)} .
$$

That is, the rms amplitude of the residual signal in the null stream is proportional to $A_{12}$. Assuming $A_{2}>A_{1}$ and that the noise levels of the two detectors are identical, the residual SNR is approximately a fraction $f_{\mathrm{s}} \times f_{\mathrm{h}}$ of the original SNR of the more sensitive detector, where

$$
f_{\mathrm{h}}=\sqrt{\frac{\sum_{t}\left(h_{+}^{2}(t)+h_{\times}^{2}(t)\right) \cos ^{2}\left(2 \psi+\xi^{\mathrm{h}}(t)+\xi_{2}\right)}{\sum_{t}\left(h_{+}^{2}(t)+h_{\times}^{2}(t)\right) \sin ^{2}\left(2 \psi+\xi^{\mathrm{h}}(t)+\xi_{2}\right)}},
$$

and

$$
f_{\mathrm{s}}=\frac{\left|\sin \left(\xi_{1}-\xi_{2}\right)\right|}{\sqrt{A_{2}^{2} / A_{1}^{2}+\cos ^{2}\left(\xi_{1}-\xi_{2}\right)}} .
$$

The quantity $f_{\mathrm{h}}$ depends on the characteristics of the wave. If the wave is such that $\sqrt{h_{+}^{2}(t)+h_{\times}^{2}(t)}$ changes more slowly with time than the phase $\xi^{\mathrm{h}}(t)$, then the average value of $f_{\mathrm{h}}$ will be approximately 1 . This will be the case for, e.g., waves of circular polarization.

For two perfectly aligned detectors, the null stream occurs at $h_{1}(t)=-h_{2}\left(t+\tau_{12}\right)$, i.e., $v=1$ due to the anti-phase arrangement of one of the arms of L1 and H1. The null stream can be constructed on a band of the sky direction corresponding to a constant (and correct) geometrical time delay. The solution for the sky direction for two nearly perfectly aligned detectors degenerates into (part of) a ring in the sky corresponding to a constant geometrical arrival time delay of the GW at the two detectors. If the source is near the null of the detectors' antenna patterns then the minimum can give an incorrect time delay.

The quantity $f_{\mathrm{s}}$ is independent of wave properties and depends only on the sky locations. We show in figure 2 an all-sky map of the value of $f_{\mathrm{s}}$ at an arbitrary time. Comparing this 


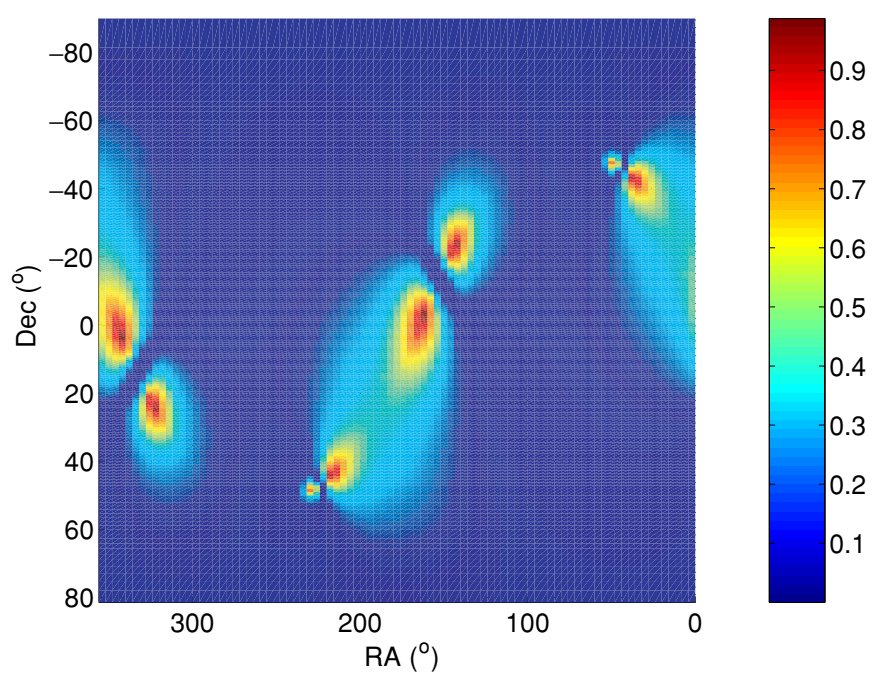

Figure 2. The all-sky map of the waveform-independent fractional SNR reduction $\left(f_{\mathrm{s}}\right)$ in the null stream of the L1-H1 network. The fraction is calculated with respect to the SNR of the more sensitive detector.

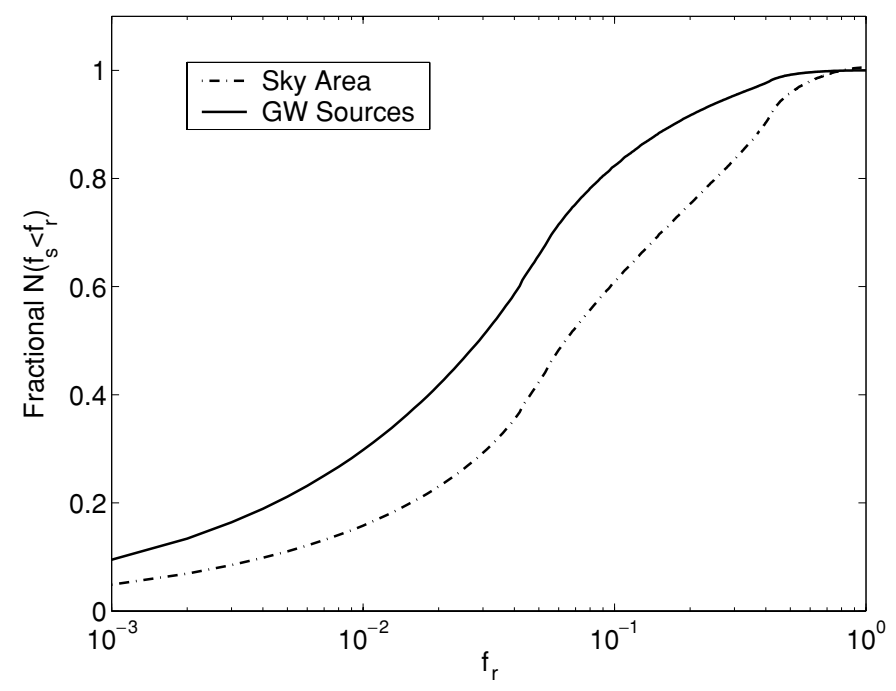

Figure 3. Number distributions in fractions of sky area and number of detectable sources as a function of the waveform-independent fractional SNR reduction $\left(f_{\mathrm{s}}\right)$ in the null stream of the L1-H1 network.

with figures 1 and 3, it is apparent that the best reduction of SNR occurs around the maximum sensitivities of the two detectors. This is expected, as $A_{12}$ can be best approximated as zero in that region. Figure 3 shows quantitatively the sky area $\left(\int \mathrm{d} \alpha \mathrm{d} \sin \delta\right)$ versus the fractional reduction $\left(f_{\mathrm{s}}\right)$. In the same figure is also plotted the corresponding fraction of the number of sources $\left(\propto \int A_{I}^{3}(\alpha, \delta) \mathrm{d} \alpha \mathrm{d} \sin \delta\right)$, assuming uniformly distributed sources in flat spacetime $\left(A_{I} \propto 1 / r\right.$, where the index $I$ refers to the more sensitive detector, $r$ is the distance to the source). It shows that, at $f_{\mathrm{h}} \sim 1,80 \%$ of sources (corresponding to $60 \%$ of the sky) can have 


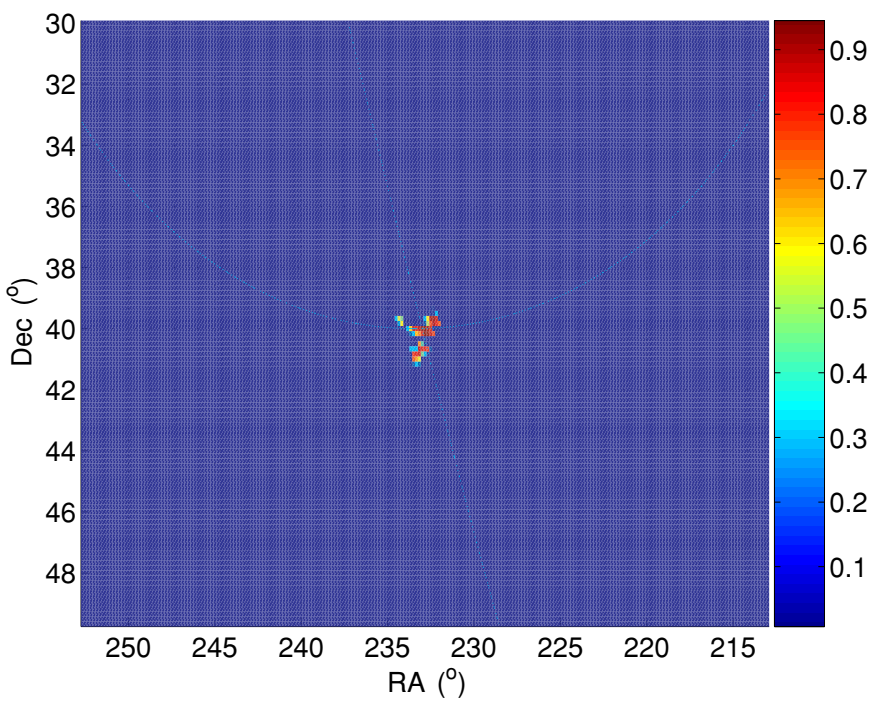

Figure 4. The sky map of the probability that the null stream statistic $P(\alpha, \delta)$ constructed for the three-detector network L1-H1-GEO follows a $\chi_{2 N}^{2}$ distribution (see text). Dotted lines indicate the correct time delay contours of the detector pairs L1-H1 and L1-GEO. The BH-BH merger source is placed at a distance $r=1 \mathrm{Mpc}$ and in a direction near the null of the L1 sensitivity (centre of the plot). The optimal three-detector SNR $=85$.

the SNR reduced by more than a factor of ten $\left(f_{\mathrm{s}}<0.1\right)$ in the constructed null stream, and that $60 \%$ of the sources can have the SNR reduced by more than a factor of $25\left(f_{\mathrm{s}}<0.04\right)$. Therefore, we can use our construction of the approximate null stream to first find sky direction with minimum fractional SNR residual, which can then be checked against the SNR residuals expected in that sky direction and checked as well for consistency with $f_{\mathrm{h}}$.

Note, however, that this may not be the optimal way of inferring sky directions. The SNR reductions do indicate the effectiveness of this approximate null stream as a veto.

\section{Implementation}

Once null data streams are constructed for each of the possible source directions for an event that passes the coincidence triggers in a burst search, we determine the sky directions that show a minimum presence of the signal, or alternatively the maximum probability that the null stream is consistent with the expected noise. If a filter of some kind has been applied to the data in order to generate the trigger, then for consistency one should presumably use this same filter on the null stream before making a decision about the presence or absence of a signal.

In this paper, we adopted a simpler test for the purpose of demonstrating the method. For each sky direction $(\alpha, \delta)$, we construct the following quantity in the (discrete) frequency domain for a given duration $T$ :

$$
P(\alpha, \delta)=2 \sum_{k=1}^{N} \frac{\left|\tilde{N}_{k}(\alpha, \delta)\right|^{2}}{\sigma_{k}^{2}},
$$

where $\tilde{N}_{k}(\alpha, \delta)$ is the Fourier transform of the null stream $N_{3}(\alpha, \delta, t), N_{2}(t)$ or $Q(\alpha, \delta, t)$ over the duration of time $T, \sigma_{k}^{2}$ is the expected variance of a true null stream at each frequency characterized by the noise spectral density $S\left(f_{k}\right), k$ is the frequency index, and $N<$ half of 


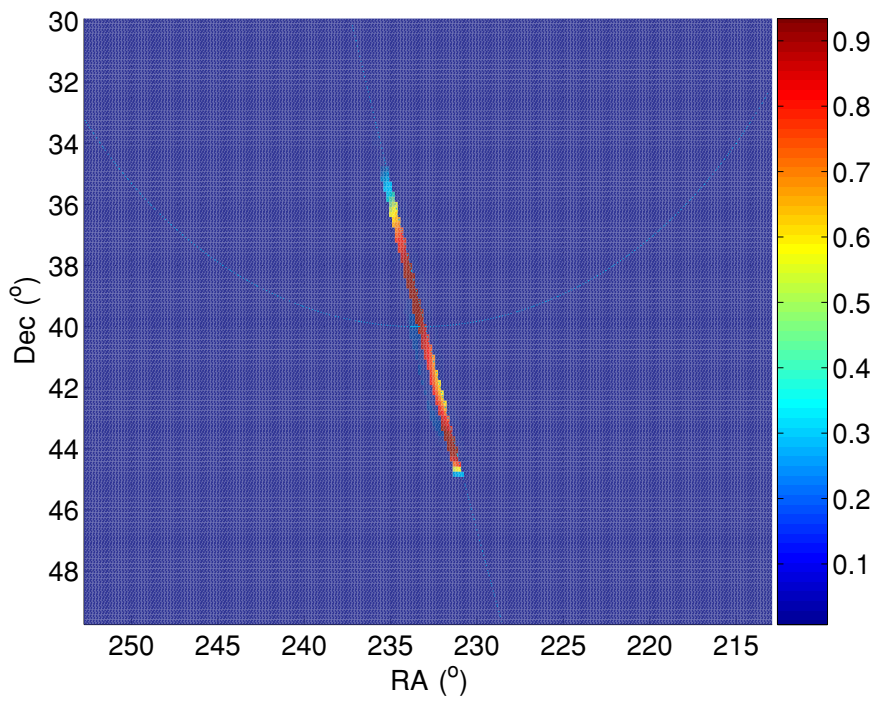

Figure 5. Same as in figure 4, but for the null-stream statistic constructed using the two-detector network L1-H1, the corresponding optimal two-detector SNR $=53$.

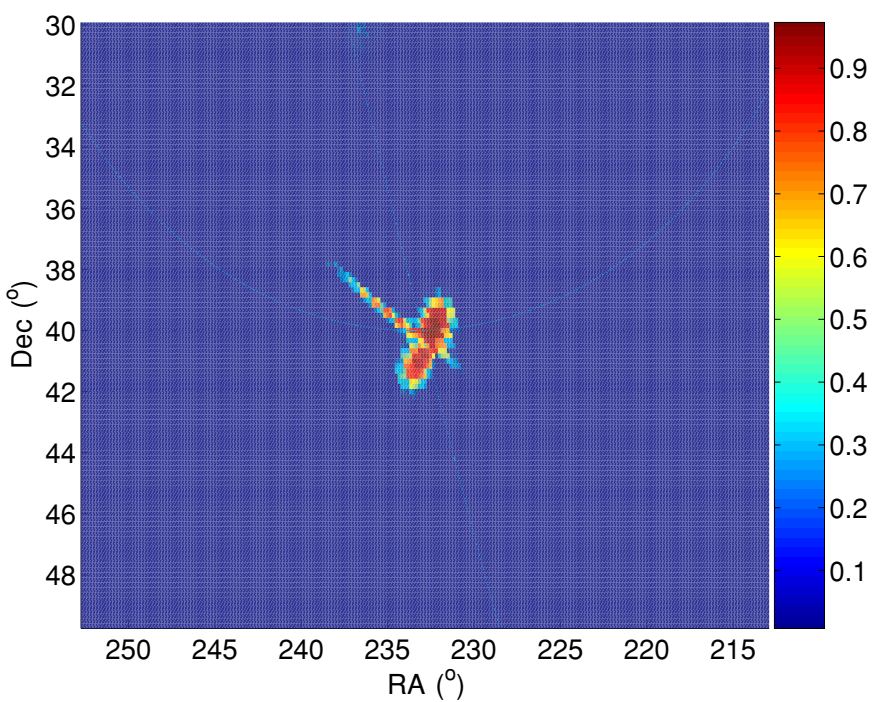

Figure 6. Same as in figure 4 for the L1-H1-GEO network, but with the source placed at $r=$ $3 \mathrm{Mpc}$. The corresponding optimal three-detector $\mathrm{SNR}=28.5$.

the number of data points. To improve the SNR, it is advantageous to have a band-limited summation in equation (17). In this paper, we sum over data points with frequencies $\leqslant 2 \mathrm{kHz}$. In a real search, the frequency bandwidth can be estimated based on initial triggers from individual detectors. For the simple $\mathrm{H} 1-\mathrm{H} 2$ null stream, $\tilde{N}_{k}(\alpha, \delta)$ is replaced with $\tilde{N}_{k}$ and searches over sky directions or time delays are not needed.

For stationary Gaussian noise, the variance of the noise in the null stream for two detectors at the same site is

$$
\sigma_{k}^{2}=\sigma_{1 k}^{2}+\sigma_{2 k}^{2}
$$




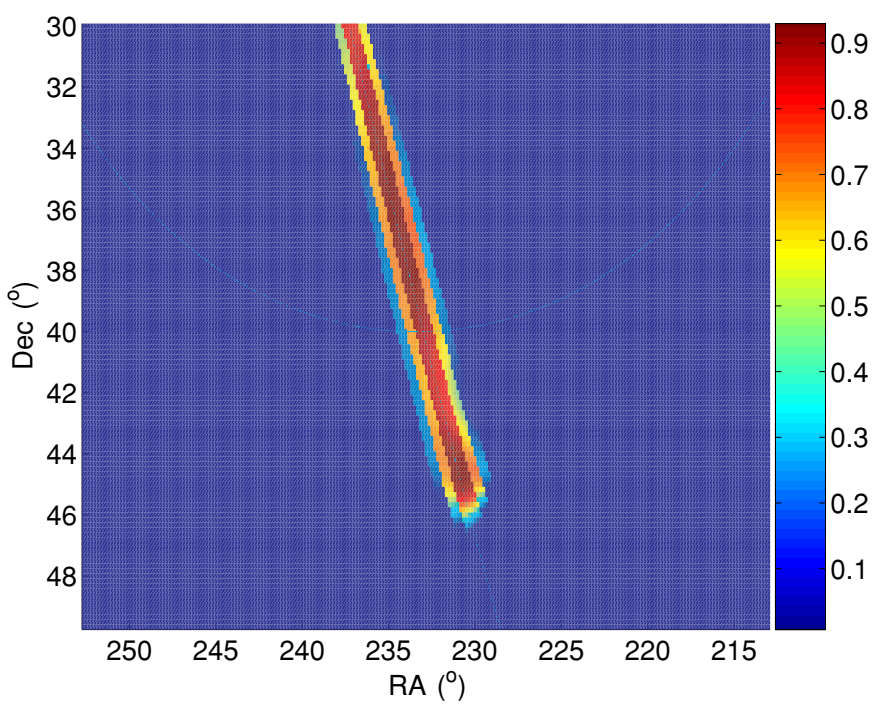

Figure 7. Same as in figure 6 but for the two-detector network L1-H1. The corresponding optimal two-detector SNR $=18$.

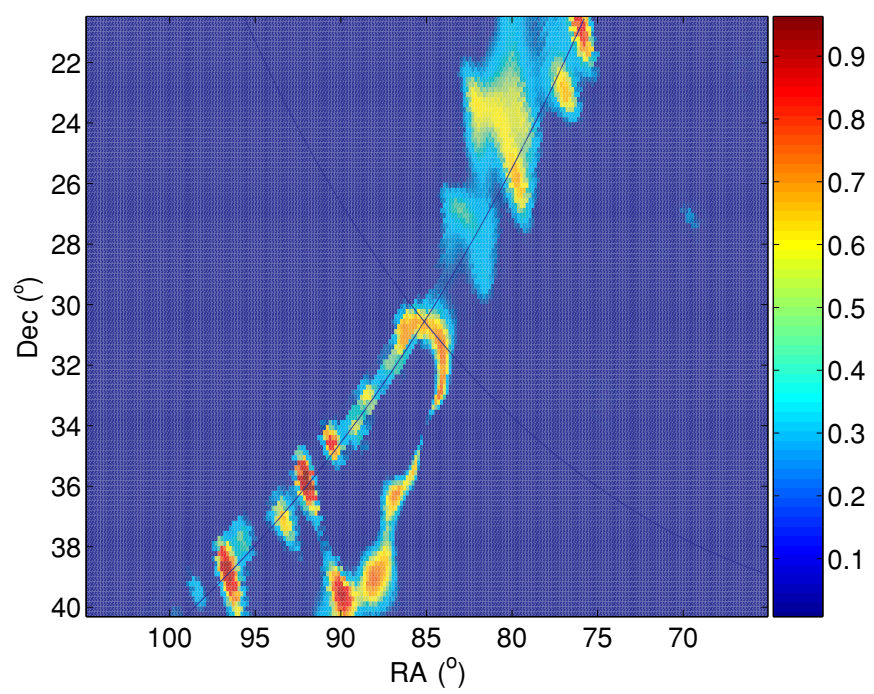

Figure 8. Same as in figure 4 for the three-detector network L1-H1-GEO, but with the source placed near the maximum L1 sensitivity (centre of the plot), source distance $r=10 \mathrm{Mpc}$, and the corresponding optimal three-detector $\mathrm{SNR}=20$, SNR in GEO is 2.

For three independent detectors, the variance is

$$
\sigma_{k}^{2}=A_{23}^{2} \sigma_{1 k}^{2}+A_{31}^{2} \sigma_{2 k}^{2}+A_{12}^{2} \sigma_{3 k}^{2},
$$

while for two nearly aligned detectors at different sites we have

$$
\sigma_{k}^{2}=A_{2}^{2} \sigma_{1 k}^{2}+A_{1}^{2} \cos ^{2}\left(\xi_{1}-\xi_{2}\right) \sigma_{2 k}^{2} .
$$

It follows that, for stationary Gaussian noise, $P(\alpha, \delta)$ is a random variable drawn from a $\chi^{2}$ distribution with $2 N$ degree of freedom. In practice, the noise distribution can be determined 


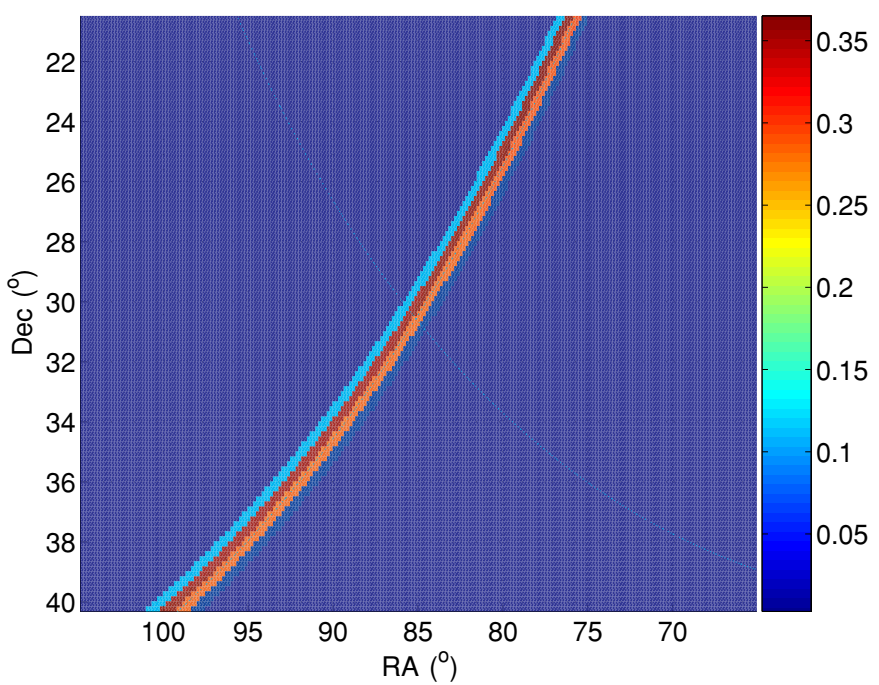

Figure 9. Same data as in figure 8, but for the two-detector network L1-H1. The corresponding optimal two-detector SNR $=20$.

from the average properties of data away from the segments we are interested in. (We adopt here a strictly frequentist approach. We leave to a later investigation the reformulation of this test in Bayesian terms.) We then search through sky directions (except for the $\mathrm{H} 1-\mathrm{H} 2$ case) for the value of $P(\alpha, \delta)$ that yields the maximum probability to be consistent with the expected noise distribution.

\section{An example application for $\mathrm{BH}-\mathrm{BH}$ merger signals}

\subsection{Simulation elements}

We choose for this simulation a signal with the two-black-hole merger waveform obtained from the Lazarus numerical relativity simulation [23]. The wave is from the merger phase of two $10 M_{\odot}$ black holes, viewed along the axis of the binary's orbital momentum. The duration of the wave is about $7 \mathrm{~ms}$ with a central frequency of about $500 \mathrm{~Hz}$. We demonstrate results from two sky directions, one near L1's maximum sensitivity and the other near its minimum sensitivity, where GEO600 is as sensitive as LIGO detectors at high frequencies despite its smaller size.

We have adopted the projected detector noise spectral densities $S(f)$ for initial LIGOs [24], and for GEO at [25] with $500 \mathrm{~Hz}$ tuning. Independent Gaussian noise samples at different times were generated according to these distributions. We obtained different SNRs by varying the distance to the source. The location information of the different $\mathrm{GW}$ observatories was obtained from [26] and references therein.

\subsection{Results}

Figures 4 and 6 show the source localization using L1, H1 and GEO. The GW source is placed at distances of 1 and $3 \mathrm{Mpc}$, at a direction near the minimum L1 sensitivity. The arrival time of the GW at L1 was chosen arbitrarily to be at $0.00 \mathrm{hr}, 18$ March 2004. The optimal SNR quoted here is defined as the optimal combination of the signal-to-noise ratios $\rho_{i}$ from the matched 


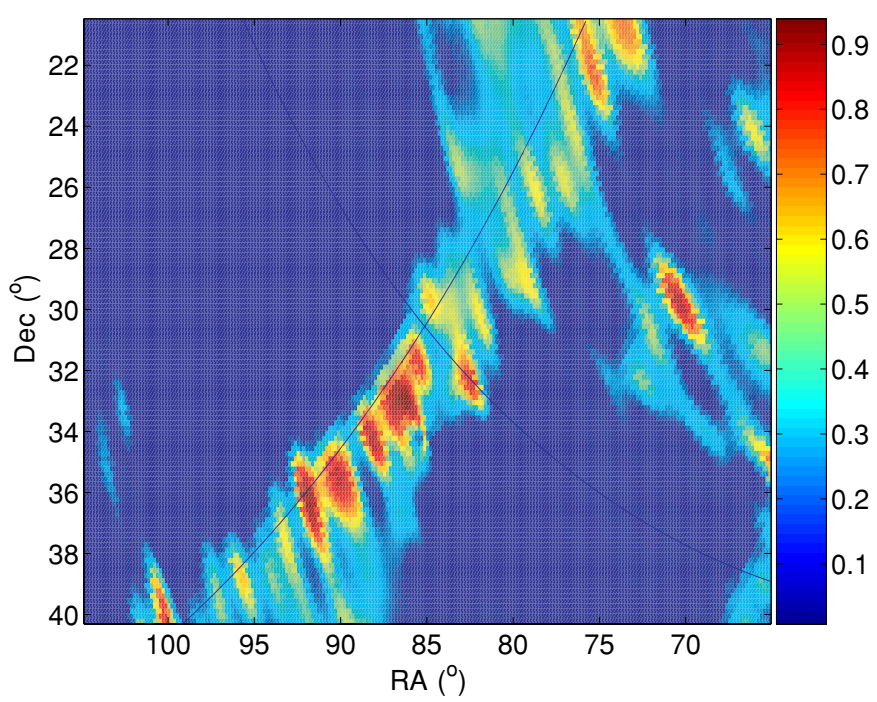

Figure 10. Same as in figure 8 for the L1-H1-GEO detector network but with the source distance $r=20 \mathrm{Mpc}$ and SNR $=10$. SNR in GEO is 1.

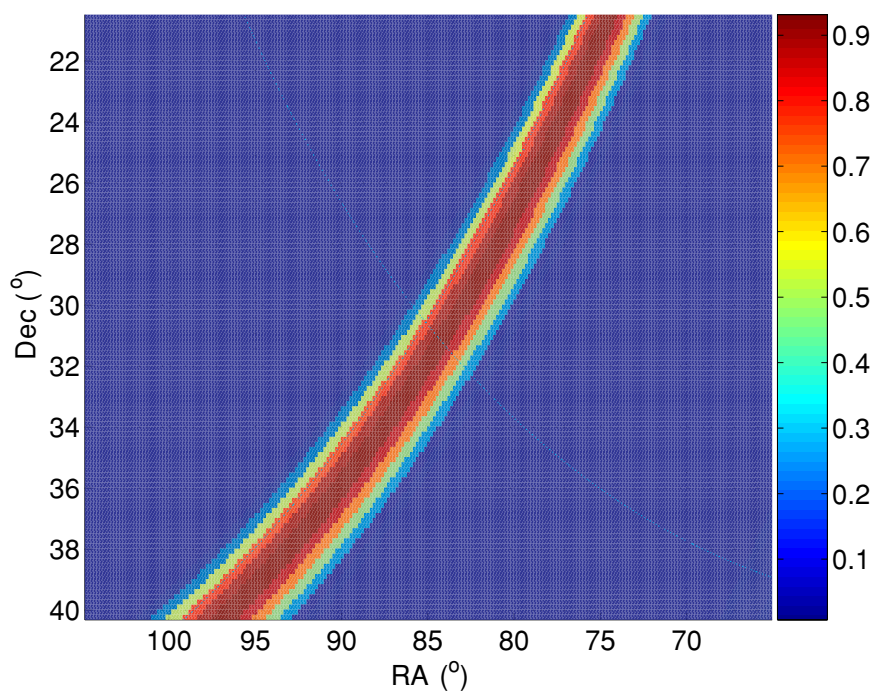

Figure 11. The same data as in figure 10 but for the two-detector network L1-H1, source distance $r=20 \mathrm{Mpc}$ and $\mathrm{SNR}=10$.

filtering technique $\rho=\sqrt{\sum_{i} \rho_{i}^{2}}$. In these two figures, $\rho=85\left(\rho_{1}=47, \rho_{2}=23, \rho_{3}=67\right)$ and 28.5. Source direction can be determined from fractions of a degree to a few degrees. Figures 5 and 7 show the results from the same data but using the two-detector network of L1 and H1 only. The optimal SNR is 53 and 18. The source direction can be determined in one dimension from fractions of a degree to several degrees.

Figures 8 and 10 show the source localization using L1, H1 and GEO but with the source placed at distances of $10,20 \mathrm{Mpc}$, in the direction near the maximum L1 sensitivity. The 
optimal SNR $\rho$ is $20\left(\rho_{1}=15, \rho_{2}=13, \rho_{3}=2\right)$ and 10. In these two cases, the SNR from GEO is around 2 and 1 . That is, GEO is not very sensitive to the signals. As a result, the spatial resolution is very poor for a three-detector network. Figures 9 and 11 show the results of the same data using L1 and H1 only (optimal SNRs are 20 and 10). The sky direction can be determined to degrees along one dimension.

Note that in the three-detector network, when the SNR is high enough, the sky location can be uniquely determined. This is an improvement on the degeneracy in localization with time delay information only.

\section{Conclusion}

We have presented the principle of constructing null streams using data from two or three detectors that are redundant or almost so. The null streams, which contain no GW signal, are useful as vetos and as localizers of source directions, as demonstrated by the examples. The source localizations can be a useful consistency check on the veto, in the sense that an event that passes the veto should also localize as expected; failure to do so might indicate other problems in the data analysis. In turn, the veto is likely to be very effective at discriminating non-Gaussian noise events from real ones. The simplest veto to use is the comparison of the two LIGO Hanford detectors, but other vetos could be used for various configurations, such as the two large LIGO detectors along with GEO600. When only the two large LIGO detectors are being used, there still seems to be an approximate null stream that is effective over most of the sky.

Note that the null stream construction method can also be used to test the validity of the theory of general relativity (GR). General relativity predicts that there are only two transverse wave polarizations in GWs $\left(h_{+}\right.$and $h_{\times}$in this context). As a result, a true null stream can be constructed for any waveforms using three detectors at different sites and the two wave polarizations can be extracted for sufficient strong signals. On the other hand, scalar-tensor theory gravitational waves can contain a transverse breathing mode in addition to these two transverse modes [27]. In this case, a null stream can be constructed for any waves with at least four detectors at different sites and the breathing mode, if present with sufficient strength, can be measured using three detectors. If the four-detector null stream shows no signal but null streams constructed from subsets of three detectors do, then this would indicate that a scalar polarization mode is present in the wave. Even if only three detectors are available, it might be possible to detect a scalar mode from the failure of the null stream, provided that the GW event can be confirmed by other means.

This paper is only a preliminary investigation of what we believe will become a powerful tool for data analysis. Many issues remain to be studied. We plan in subsequent publications to address: (1) use of the veto from $\mathrm{H} 1$ and $\mathrm{H} 2$ in realistic data analysis, taking into account calibration error, spatial and timing resolution, and the effect of nonlinear trigger algorithms; (2) understanding under what circumstances the H1-L1-GEO veto will be useful and whether the null stream will help localize, and the effect of including the VIRGO detector in threeand four-detector null streams, and investigating practical issues such as the best use of information on time delays and signal durations; (3) consideration of incorporating the nullstream search algorithm into an all-sky coherent detection method by adding data together to maximize SNRs at the same sky location where null streams are constructed (one method already proposed in $[12,28]$ ); (4) signal recovery and parameter estimation; (5) computational cost and search grid size; and (6) application to testing alternative theories for gravitational waves. 


\section{Acknowledgments}

We would like to thank Peter Saulson for carefully reading through our manuscript and for his critical comments. We also thank B S Sathyaprakash for very useful and inspiring discussions on this work.

\section{References}

[1] Tinto M, Armstrong J W and Estabrook F B 2000 Phys. Rev. D 63021101

[2] Tinto M and Larson S L 2004 Phys. Rev. D 70062002

[3] Shawhan P 2004 Talk presented at 9th Gravitational Wave Data Analysis Workshop, Annecy, France, 15-18 December 2004

[4] Hewitson M 2005 Class. Quantum Grav. 22 S891

[5] Ando M 2005 Class. Quantum Grav. 22 S1283

[6] Acernese F et al 2005 Class. Quantum Grav. 22 S869

[7] Abbott B et al 2004 Phys. Rev. D 69102001

[8] Abbott B et al 2004 Phys. Rev. D 69082004

[9] Abbott B et al 2004 Phys. Rev. D 69122001

[10] Abbott B et al 2004 Phys. Rev. D 69122004

[11] Abbott B et al 2005 Phys. Rev. Lett. 94181103 (Preprint gr-qc/0410007)

[12] Gürsel Y and Tinto M 1989 Phys. Rev. D 403884

[13] Jaranowski P and Krolak A 1994 Phys. Rev. D 491723

[14] Bose S, Dhurandhar S V and Pai A 1999 Pramana 1125

[15] Pai A, Dhurandhar S and Bose S 2001 Phys. Rev. D 64042004

[16] Finn L 2001 Phys. Rev. D 63102001

[17] Sutton P J 2004 Class Quantum Grav. 21 S1801

[18] Cadonati L 2004 Class Quantum Grav. 21 S1695

[19] Rakhmanov M and Klimenko S 2005 Class. Quantum Grav. 22 S1311

[20] Jaranowski P, Krolak A and Schutz B 1998 Phys. Rev. D 58063001

[21] Fafone V 2004 Class. Quantum Grav. 21 S377

[22] Abramovici A et al 1992 Science 256325

[23] Baker J, Campanelli M, Louston C O and Takahashi R 2002 Phys. Rev. D 65124012 (Preprint astro-ph/0202469)

[24] Lazzarini A et al 1996 LIGO Science Requirement Documents LIGO E950018-02, and http://www.ligo. caltech.edu/lazz/distribution/LSC_Data/srd.html

[25] http://www.aei.mpg.de/jrsmith/geocurves.html

[26] Allen B 1996 Preprint gr-qc/9607075

[27] Will C M 2001 Living Rev. Rel. 44 (online article): cited on 10 April 2005, http://www.livingreviews.org/ 1rr-2001-4

[28] Anderson W G, Brady P R, Creighton J D E and Flanagan E E 2001 Phys. Rev. D 63042003 